\title{
The dynamic scheduling algorithm based on heuristic rule
}

\author{
Yan $\mathrm{Ge}^{1, \mathrm{a}}$, Aimin Wang ${ }^{1, \mathrm{~b}}$, Jincheng Cheng ${ }^{1, \mathrm{c}}$ \\ ${ }^{1}$ Engineering,Beijing Institute of Technology, Beijing 100081,China \\ ageyan_1993@163.com, bwangam@bit.edu.cn, c493118224@qq.com
}

Keywords: spatial scheduling; algorithm; punctuality; efficiency; batch rule; greedy rule;

Abstract. To deal with the problems in shipbuilding spatial scheduling with rectangular workplace and rectangular blocks, a dynamic scheduling algorithm based on heuristic rule was put forward. The algorithm consists of two rules to achieve the multi-day scheduling and guarantees the punctuality of blocks and the efficiency of the workplace at the same time. The batch rule was proposed to segment the blocks in batches according to their earliest start time. The greedy rule based on dynamic programming was put forward to determine the location of the blocks in the field and maximize the efficiency of the workplace. Finally, a program was developed with the actual data of a shipyard to verify the feasibility and effectiveness of the algorithm. The result shows that the algorithm obtains satisfactory results in all indexes mentioned above.

\section{Introduction}

Shipbuilding industry is an important pillar of the national economy, but as the core of the ship shell construction, blocks production has not been able to achieve efficient automation since blocks are always very heavy and hard to be fitted and welded. Spatial scheduling belongs to the typical spatio-temporal coupling scheduling problem because it considers not only the layout of current blocks, but also the replacement between blocks in and blocks out[1]. The application of mature tools in actual production of blocks is less, mostly, workers make a one-time layout rely on their experience or the rectangular magnetic film placed on a whiteboard[2]. This method can't improve the efficiency, and it is difficult to perform an ongoing plan of spatial scheduling. In view of the complexity of this problem, an algorithm consists of batch rule and greedy rule based on dynamic programming has been proposed to achieve the multi-day scheduling and guarantee the punctuality of blocks and the efficiency of the workplace at the same time in this paper.

Aiming at the problem of block spatial scheduling, Lee et.al[3] sort the blocks by their relaxation time and then arrange the blocks to the workplace in order. They get a high efficiency with this method. A heuristic rule which put blocks into batches to Shorten the completion time was proposed by Sung et.al[4]. Park et.al[5] has proposed the heuristic rule based on finding the arrangement points. Ranjan[6] et.al achieved the maximization of space utilization with the application of the left-most and bottom-most rule. But the rule didn't take the large waste arisen from the field segmentation due to the discrete completion time of blocks into account. Zhiying Zhang et.al[7] simplified the workplace to a strip shape to change the plane-time three-dimensional problem into length-time two-dimensional problem, but this algorithm will lead to the irrational use in the longitudinal direction of the field and some blocks can't be arranged in field on time.

The algorithms above all solved the problem and achieved a high utilization of the workplace in a certain extent, indicating that heuristic rules have considerable advantages in solving this kind of problem. Based on the above work, a dynamic scheduling algorithm consists of batch rule and greedy rule has been proposed aiming at the efficiency of the workplace and punctuality of blocks. 


\section{The spatial scheduling problem[8]}

This paper considers the spatial scheduling problem with rectangular blocks and rectangular workplace. The problem can be described as: the $\mathrm{N}$ blocks should be arranged in a suitable position in the workplace with a specific sequence in the specified time, and the scheduling scheme makes the workplace have a high efficiency. Here is the mathematical model of the problem:

$$
\begin{aligned}
& \max : \eta=\sum S_{i} / S_{W P} \times 100 \% \\
& b_{i} \cap W P=b_{i}, i=1,2,3 \cdots, \mathrm{N} \\
& b_{i} \cap b_{j}=\varnothing, i 、 j=1,2,3 \cdots, \mathrm{N}, i \neq j \\
& D i \geq t_{E S T i}, i=1,2,3 \cdots, \mathrm{N}
\end{aligned}
$$

$S_{i}$ and $S_{W P}$ are the total area of blocks in the workplace and the area of the workplace. $b_{i}$ represents a block and WP represents the workplace. $D i$ and $t_{E S T i}$ are the actual start time and the earliest start time of a block.

\section{The dynamic scheduling algorithm based on heuristic rule}

The whole structure of the algorithm is shown in Fig.1.

From the diagram, we can see that there are two key technologies in the algorithm. The greedy rule corresponds to the objective function of high efficiency and the constraint of collision. The batch rule is used to select the first batch of blocks to enter the workplace according to the earliest start time and the remaining area to satisfy the constraint of punctuality of blocks.

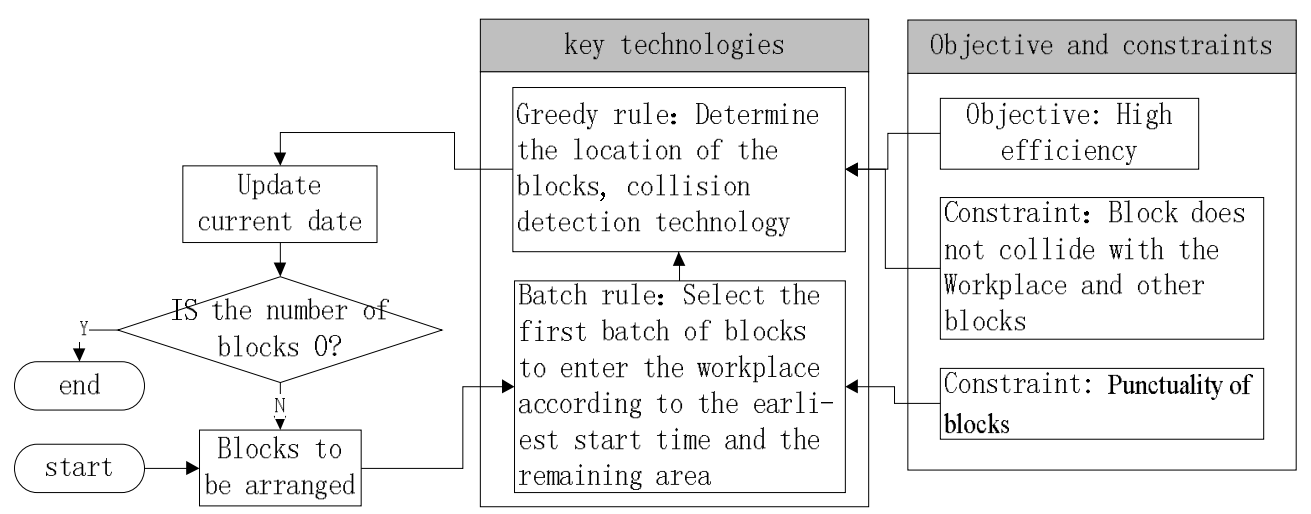

\section{The batch rule}

Fig. 1 The whole structure of the algorithm

The batch rule is used to select the first number of blocks to enter the workplace from the blocks that have not been arranged. The bases for the rule are the earliest start time of blocks and the remaining area of the workplace. First, the rule calculates the area of the remaining workplace. Then the remaining blocks are sorted according to their earliest start time. Finally a batch of blocks are selected. For instance, at the current juncture, the date is datenow, the number of the remaining blocks is $N$, the area of the remaining workplace is $S_{r e}$. Based on the given data, the batch rule 
selects $n$ of $N$ blocks to enter the workplace. $n$ meets the following relationship:

$$
\sum_{i=1}^{n} S_{b i} \geq S_{r e} \times p, \sum_{i=1}^{n-1} S_{b i}<S_{r e} \times p
$$

Expression (1)

In expression (1), $S_{b i}$ is for the area of the i-th block, $p$ is for a factor about the waste of the workplace. If we will select the $\mathrm{j}$-th batch of blocks, then the value of $p$ satisfies the following relationship:

$$
p=90 \%-j \times 3 \%
$$

Expression (2)

\section{The greedy rule}

The greedy rule determines the location of the blocks. The main steps of the rule are:

(1)Make the batch of blocks which selected by the batch rule the current pending blocks. The interval of the earliest start time of blocks is $\left[D_{s}, D_{e}\right]$ and the earliest start time of the remaining

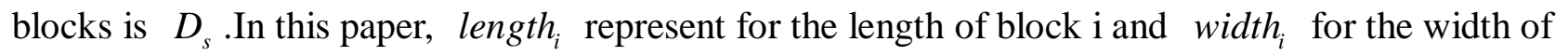
block $i$. The length and the width of the workplace is $L$ and $W$;

(2)For the blocks which will be arranged in, the method of dynamic programming is used to find block groups to satisfy expression (3) and expression (4). In expression (3), num is the number of blocks in each block group and $\overline{\text { width }}$ is the average of all blocks.

$$
\begin{array}{cc}
\text { num }=\operatorname{int}\left(W / \frac{1}{\text { width }}\right) & \text { Expression (3) } \\
0 \leq W-\sum_{m=1}^{\text {num }} \text { width }_{m} \leq W \times 5 \% & \text { Expression (4) }
\end{array}
$$

Expression (4) finds the block groups which can match against the workplace on the width direction. It ensures the full use of the workplace in the longitudinal direction. Expression (3) keeps narrow blocks to fill the gap and avoids them addition to the block groups.

(3)The block groups we have found in step (2) can be divided into the following categories:

(1) The earliest end times of all blocks are not later than $D_{s}$, which is shown in Fig.2(a);

(2)The earliest end times of all blocks are later than $D_{s}$, which is shown in Fig.2(c);

(3)The earliest end times of some blocks are later than $D_{s}$ and some are not later than $D_{s}$, which is shown in Fig.2(b);

In this case, the value of $D_{s}$.is 16 .

Block groups as shown in Fig.2 (a) should be placed close to the left edge of the workplace and block groups as shown in Fig. 2 (c) should be placed close to the right edge of the workplace. For block groups as shown in Fig.2 (b), there are two kinds of situations. If most blocks in the block group have a large earliest end time, this block group should be placed close to the right edge of the workplace. Else it should be put close to the left edge of the workplace. Fig. 3 shows positions of 
block groups shown in Fig.2.

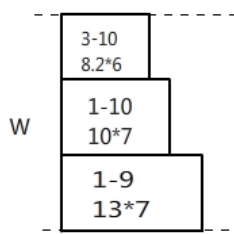

(a)

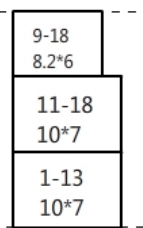

(b)

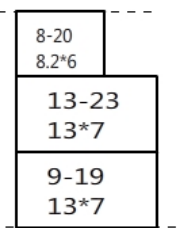

(c)

Fig. 2 Three categories of block groups

(4) Arrangement of fragmented blocks:

(1)First, sort the blocks according to their length;

(2) Select blocks in the sequence into the workplace successively. If the earliest end time of a

\begin{tabular}{|c|c|c|}
\hline $\begin{array}{l}3-10 \\
8.2^{*} 6\end{array}$ & & $\begin{array}{l}8-20 \\
8.2^{*} 6\end{array}$ \\
\hline $\begin{array}{l}1-10 \\
10 * 7\end{array}$ & $\begin{array}{l}11-18 \\
10 * 7\end{array}$ & $\begin{array}{l}13-23 \\
13 * 7\end{array}$ \\
\hline $\begin{array}{l}1-9 \\
13 * 7\end{array}$ & $\begin{array}{l}1-13 \\
10 * 7\end{array}$ & $\begin{array}{l}9-19 \\
13 * 7\end{array}$ \\
\hline
\end{tabular}

Fig. 3 Positions of block groups block is later than $D_{s}$, put it to the right and bottom corner of the workplace. Else put it to the left and bottom corner of the workplace;

(3)Detect collision for the block. The detection steps are:

a Judge whether all the vertices of the current blocks are in the workplace;

b Judge whether all the vertices of the current blocks are in the contour of the other blocks.

If the above two conditions are satisfied, the blocks collision won't happen, else the blocks will collide against the boundary of workplace or the existing blocks. Obviously, the key point of the detection is to judge whether the point is inside the rectangular or not.

If the collision occurs, move the block to the secondary right(left) and bottom corner of the workplace until a feasible placement scheme is produced;

(4)Arrange all blocks according to (2)and (3);

(5) When the next batch of blocks enter the workplace, the rule of arrangement is the same as step (3) and step (4).

Above is the whole content of the greedy rule. The mind of dynamic programming is used to find block groups in the rule. It makes the longitudinal direction of the workplace be fully utilized. The greedy arrangement of blocks ensures the maximum efficiency.

\section{Simulation experiment}

According to the dynamic scheduling algorithm based on heuristic rule proposed above, a simulation example has been performed in VS2010. The data of blocks and workplace are collected in the actual data of a shipyard in Shanghai. The number of blocks is 35, the end time of the task is the 27 th day, the length of the workplace is $50 \mathrm{~m}$ and the width is $20 \mathrm{~m}$. Results of partial batches are shown in Fig.4.

\begin{tabular}{|c|c|c|c|c|c|c|}
\hline $\begin{array}{l}3-10 \\
8.2^{*} 6 \\
\end{array}$ & $\begin{array}{l}1-1 \\
10^{*} \\
\end{array}$ & & & $\begin{array}{l}12-25 \\
8.2^{*} 6 \\
\end{array}$ & $\begin{array}{l}9-18 \\
8.2^{* 6} \\
\end{array}$ & $\begin{array}{l}8-20 \\
8.2^{*} 6 \\
\end{array}$ \\
\hline $\begin{array}{l}1-10 \\
10 * 7\end{array}$ & 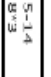 & 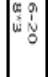 & $\begin{array}{l}14-23 \\
10 * 7\end{array}$ & $\begin{array}{l}11-18 \\
10 * 7\end{array}$ & & $\begin{array}{l}13-23 \\
13 * 7\end{array}$ \\
\hline $\begin{array}{l}1-9 \\
13^{* 7}\end{array}$ & & & $\begin{array}{l}-20 \\
3 \times 3.3 \\
15+3.3 \\
.8+3.3\end{array}$ & $\begin{array}{l}1-13 \\
10 * 7\end{array}$ & & $\begin{array}{l}9-19 \\
13^{\star} 7\end{array}$ \\
\hline
\end{tabular}

(a)

\begin{tabular}{|c|c|c|c|c|c|c|}
\hline \multirow{2}{*}{ 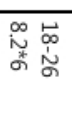 } & 17 & & $\begin{array}{l}18-26 \\
8.2^{*} 6\end{array}$ & $\begin{array}{l}12-25 \\
8.2^{*} 6 \\
\end{array}$ & $\begin{array}{l}9-18 \\
8.2^{*} 6 \\
\end{array}$ & $\begin{array}{l}8-20 \\
8.2^{*} 6 \\
\end{array}$ \\
\hline & $7.8^{\star} 6.8$ & 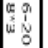 & $14-23$ & \multirow{2}{*}{$\begin{array}{l}11-18 \\
10 * 7\end{array}$} & \multirow{2}{*}{\multicolumn{2}{|c|}{$\begin{array}{l}13-23 \\
13 * 7\end{array}$}} \\
\hline \multirow{3}{*}{\multicolumn{2}{|c|}{$\begin{array}{l}18-26 \\
10.3^{*} 9.4\end{array}$}} & & $10 * 7$ & & & \\
\hline & & & $\begin{array}{l}1-20 \\
3.3 \times 3.3\end{array}$ & \multirow{2}{*}{$\begin{array}{l}18-28 \\
10 * 6\end{array}$} & \multicolumn{2}{|r|}{ 9-19 } \\
\hline & & & 3.27 & & & $13 \star 7$ \\
\hline
\end{tabular}

(b)

Fig. 4 Results of partial batches

Fig.4 (a) shows the scheduling result of the first batch of blocks. The interval of the earliest start time of blocks is [1,14]; Fig.4 (b) shows the scheduling result of the second batch of blocks. 
The interval of the earliest start time of blocks is $[16,18]$. Data statistics for scheduling results are shown in Table 1.

Table 1 Results of scheduling

\begin{tabular}{|c|c|c|}
\hline Batches & $\begin{array}{c}\text { Number of } \\
\text { delay blocks }\end{array}$ & Efficiency \\
\hline 1 & 0 & 94.413 \\
\hline 2 & 0 & 89.129 \\
\hline 3 & 0 & 87.979 \\
\hline 4 & 0 & 81.682 \\
\hline
\end{tabular}

What can be concluded from Fig. 4 and Table 1 are:

(1)The four efficiencies are all higher than the result of traditional scheduling which is about $70 \%$;

(2)With the previous batch of blocks to leave and the next batch to enter, the remaining workplace becomes more and more irregular, which increases the difficulty of the placement. Therefore, The efficiency of each batch has a certain degree of reduction compared to the previous batch;

(3)There is no block delayed in all batches. It shows that the batch rule proposed in this paper is feasible and effective in the case of a small number of blocks.

\section{Conclusions}

(1) Aiming at multi-days continuous shipbuilding blocks spatial scheduling problem with rectangular blocks and rectangular workplace, the objective function and constraints model considering the optimal completion time and efficiency are established, and the dynamic scheduling algorithm based on heuristic rule is proposed.

(2) The batch rule is proposed based on the earliest start time of blocks and the remaining area of the workplace. It takes into account the placement of multiple time periods, to avoid the messy position distribution caused by blocks moving into the workplace continuously.

(3) The greedy rule based on greedy algorithm and dynamic programming is proposed. Dynamic programming makes the longitudinal direction of the workplace be fully utilized, and the greedy arrangement of blocks ensures the maximum efficiency.

(4) In combination with the actual data of a shipyard in Shanghai and the algorithm implementation, the verification shows that the proposed method in this paper can effectively solve the practical spatial scheduling problem, reduce the waste of workplace and ensure the punctuality of blocks.

\section{References}

[1] ZHANG zhiying, YANG kaike, YU jinwei, CHEN qiang. Improved particle swarm optimization algorithm for dynamic spatial scheduling[J]. Journal of Harbin Engineering University, 2009,30(12):1344-1350(in Chinese).

[2] ZHENG junli, CHEN feng, JIANG zhibin, CHEN qiang. Algorithm for reducing the maximum completion time for shipbuilding spatial scheduling[J]. Journal of Shanghai Jiaotong University,2009,43(4):663-668(in Chinese).

[3] LEE K J, LEE J K, CHOI S Y. A Spatial Scheduling System and its Application to Shipbuilding:DAS-CURVE[J]. Expert Systems with Applications, 1996,10(3-4):311-324. 
[4] Sung C S, Choung Y I. Minimizing makespan on a single burn-in oven semiconductor manufacturing[J]. European Journal of Operational Research 2000,120,:559-574.

[5] Park K, LEE K, Park S, et al. Modeling and solving the spatial block scheduling problem in a shipbuilding company[J]. Computer ind Engng,1996,30(3):357-364.

[6] Ranjan V, Duck Y Y. Dynamic spatial block arrangement scheduling in shipbuilding industry using genetic algorithm[C]//2005 $3^{\text {rd }}$ IEEE International Conference on Industrial Informatics. Indin: IEEE,2005,444-449.

[7] ZHANG zhiying, WANG lei, JIANG hanbin, LI zhen. Spatial scheduling meshod for hull block construction in shipbuilding[J]. Journal of Shanghai Jiaotong University, 2008,42(4): 651-656(in Chinese)

[8] BO LI,ZHI-YAN ZHAO,GANG LI. A dynamic scheduling method for spatial layout planning. $[C] / /$ Proceedings of the Fourth International Conference on Machine Learning and Cybernetics. Indin: IEEE,2005,3612-3617. 